\title{
El papel de la participación en la transición socio-ecológica de la ciudad
}

\section{The role of citizen participation in the socio-ecological transition of the city}

Dra. Marta Donadei es investigadora del Grupo ADICI (HUM-810) y Asistente Honoraria del Departamento de Urbanística y Ordenación del Territorio de la ETSA Sevilla (España) (donadei.marta@gmail.com) (https://orcid.org/0000-0003-1620-1459)

\begin{abstract}
Resumen
Pese a la creciente sensibilización a los efectos del cambio climático, sus cada vez más acusadas consecuencias demuestran el peligro que supone el seguir con el imperativo unilateral del crecimiento económico, caracterizado por una «intoxicación consumista» que nos ha llevado a esta situación de carestía crónica global de recursos. En este contexto, el nuevo enfoque no puede reducirse a mero arreglo cosmético de un paradigma en crisis, sino que requiere también un cambio de paradigma para avanzar hacia la sostenibilidad ambiental y la equidad social. Para ello se quiere incidir en el hecho de que la involucración activa de la población resulta elemento clave para activar un cambio en la forma que la sociedad occidental tiene de entender la naturaleza y para construir alternativas reales al actual modelo de ocupación del territorio, que estén basadas en el paradigma ecológico. Así, el planteamiento de este artículo encuentra sus bases teóricas en la Producción y Gestión Social del Hábitat (en adelante PGSH), entendiendo por Trasformación Social del Hábitat el conjunto de procesos tendientes a la transformación del hábitat existente en los cuales la iniciativa ciudadana tiene un papel activo en la toma de decisiones. A través de esta revisión se concluye que la participación no sólo puede ayudar a la transformación de las condiciones materiales de la transición ecológica de la ciudad, sino que, a partir de un trabajo profundo que requiere grandes esfuerzos por parte de todos los grupos de actores involucrados, permite generar un proceso en el que ciudadanos, políticos y técnicos maduren colectivamente.
\end{abstract}

\begin{abstract}
Despite the growing sensitivity about the effects of climate change, its marked consequences show the risk of continuing with the unilateral imperative of economic growth, characterized by a "consumer intoxication" that has driven this society to the current situation of global chronic lack of resources. In this context, the new approach cannot be reduced to a mere cosmetic repair of a paradigm in crisis" but it needs a change of paradigm to advance towards environmental sustainability and social equity. Therefore, it is important to focus on the fact that a population that is actively involved is a key element in order to activate a change in the way occidental society has to understand nature, and to build real alternatives to the current model of territory occupation based on the ecological paradigm. The approach of this article has its theoretical bases on the Social Production and Management of Habitat (henceforth PGSH), understanding by Social Transformation of Habitat those processes that tend to transform the existing habitat in which citizen initiative has an active role in the decision making. Thus, it is concluded that participation cannot only help transforming the material conditions of the ecological transition of the city, but, from a profound work that requires great efforts by all stakeholder groups involved, it allows to generate a process in which citizens, politicians and technicians grow up and mature collectively.
\end{abstract}

\section{Palabras clave I keywords}

Participación social, investigación social, urbanismo, transición socio-ecológica, iap.

Social participation, social research, urban planning, socio-ecological transition, par.

Cómo citar: Donadei, M. (2019). El papel de la participación en la transición socio-ecológica de la ciudad. Retos Revista de Ciencias de la Administración y Economía, 9(17), 55-70. https:// doi.org/10.17163/ret.n17.2019.04 


\section{Introducción}

Si se asume la definición de la arquitectura como "conjunto de las modificaciones y alteraciones introducidas sobre la superficie de la Tierra de acuerdo con las necesidades humanas, exceptuando únicamente el riguroso desierto" (Morris 1981 en Benevolo, 1979 , p. 15), el concepto de arquitectura nos sitúa en el campo de tensiones entre naturaleza y cultura, y encuentra sus raíces en las necesidades humanas. Así, la arquitectura como tal, y por consiguiente el urbanismo, no pueden ser considerados solo «cosas de arquitectos» sino que entran en una dimensión más grande: la del Hábitat Social.

Pelli $(2010 ; 2007)$ define el Hábitat Social como el sistema de situaciones físicas, sociales, económicas, jurídicas, políticas, ambientales y simbólicas entre las que, aplicando los conceptos de la «teoría de la complejidad» de Morin (1994), se encuentran relaciones recursivas y complementarias (De Manuel Jerez, 2010). En este sentido "cualquier acto de producción, eliminación o conservación de una parte o componente del hábitat modifica el equilibrio, el funcionamiento y la calidad de todo el conjunto y afecta los de otros componentes, existentes o futuros" (Pelli, 2010, p.41).

Pero el Hábitat Social es sólo un subsistema del ecosistema planetario con el que interactúa y al que transforma: «las reglas del juego económico/financiero dominante» (Naredo, 2000), caracterizado por una obsesión por el crecimiento y que está basado en la progresiva explotación y uso masivo de los recursos naturales, que han provocado un cada vez mayor deterioro territorial y demuestran que ya hemos desbordado ampliamente los límites de regeneración del planeta (Emmott, 2013; Meadows, Randers, \& Meadows, 2006).

En este contexto, las acusadas desigualdades sociales y la cada día más alarmante crisis ecológica muestran el fracaso del modelo occidental y ponen en crisis el mismo postulado del crecimiento, demostrando que es necesario activar una deconstrucción del pensamiento económico imperante, replanteando el lugar de la economía para verla como simple medio de vida y no como fin último (Castiblanco, 2008; Common \& Stagl, 2008; Georgescu-Roegen, 1971; Naredo, 2011) y revisando en profundidad los actuales modelos urbanos y territoriales, para avanzar hacia la equidad social y la recuperación de los equilibrios naturales y territoriales, por medio de la reducción ecuánime del despilfarro, de la sobreproducción y del sobreconsumo, garantizando a la vez el aumento del bienestar social (Caravaca Llamas, 2012; Latouche, 2009; Marcellesi, 2010; Taibo, 2009).

Cualquier pauta social y cultural, desde las cuestiones privadas de cada ser hasta la organización político-económica de una sociedad, tiene una repercusión, directa o indirecta, en el medio ambiente. Por lo tanto, es esencial trabajar con la dimensión cultural de la sostenibilidad porque es la cultura la que nos hace actuar en el territorio de una forma u otra, contribuyendo a construir un modelo basado en el deterioro de los recursos o fomentando patrones de vida sostenible bajo un enfoque ecointegrador.

En este sentido, la participación se convierte en elemento clave para poder activar la transición socio-ecológica de nuestras ciudades y territorios (García Montes, 2018): si no se replantean las formas de consumo en nuestro día a día, como ciudadanos, profesionales, empresas, sociedades, etc., las nuevas facilidades y soluciones tecnológicas -a menudo vistas como la solución a cualquier tipo de problema- nos 
llevarán, por el contrario, a derroches cada vez más insensatos («paradoja de Jevons», "efecto rebote»). Así, "necesitamos un sistema ético en el cual el mundo natural tenga valor no sólo en cuanto útil para el bienestar humano, sino por sí mismo”, porque formamos parte de él (Lovelock, 2006, p. 214).

Todo ello supone, por una parte, la generación de políticas más acordes con el buen gobierno del territorio, y por otra la necesidad de plantear nuevas formas de planificar mucho más flexibles y dinámicas para que puedan adaptarse al ritmo de las transformaciones intensas y rápidas que se dan en el uso del suelo, y que están inducidas por factores socio-económicos. Pero al mismo tiempo es necesario fomentar estrategias orientadas al cambio en el pensamiento y en los hábitos de vida y consumo de la sociedad, para sustituir los actuales valores individualistas y cumulativos, pero considerando al mismo tiempo que las personas son agentes activos de ese cambio.

Cualquier intento de imposición de proyectos basados en nuevos esquemas desarrollados exógenamente genera sentimientos y actitudes de rechazo por parte de la población (Díaz Rodríguez \& Rodríguez Darias, 2012) y estará destinado al fracaso. Por el contrario, son numerosas las iniciativas sociales que reclaman un protagonismo en la toma de decisiones sobre el hábitat, en particular frente al urbanismo convencional planificado y regulado (arriba-abajo). En los últimos años han proliferado movimientos que reivindican su derecho a participar «desde abajo» en la transformación urbana, para una mejora del entorno próximo que afecta al desarrollo de la vida cotidiana y que se desarrolla a partir de la auto-organización entre ciudadanos junto a colectivos profesionales (Freire, 2009; González-Arriero, 2017).

Frente a esta dicotomía entre los modelos planificados del urbanismo tradicional y las crecientes iniciativas ciudadanas que reclaman su protagonismo y autonomía en la toma de decisiones, se quiere demostrar la necesidad de:

[...] construir herramientas de conocimiento y estrategias de acción que permitan comprender y actuar ante problemáticas típicamente complejas, [...] que escapan a la fragmentación del conocimiento en disciplinas estancas. Y herramientas que inserten el conocimiento técnico en procesos transformadores que recuperen la POLIS, los espacios de participación de los ciudadanos en la toma de decisiones (Cambil Medina et al., 2006, p. 1).

\section{Respuestas locales a fenómenos globales: hacia un nuevo urbanismo}

Las sociedades occidentales han afrontado transformaciones radicales a lo largo del último siglo de historia, caracterizadas por una constante y cada vez más célere innovación tecnológica. Dicha innovación ha dado paso a una profunda globalización que sigue generando grandes impactos induciendo cambios progresivos (tanto positivos como negativos, y de orden material y simbólico) en su estructura política, económica, cultural y territorial. En particular, el actual paradigma tecnológico ha generado una segmentación y descentralización de la producción cada vez más grandes, para poder aprovechar las diferentes potencialidades ofrecidas por los distintos territorios. De esa forma se ha desarrollado una competencia cada vez mayor entre empresas, sectores y territorios siguiendo procesos de diferente tipo que han ido conformando $\mathrm{y}$ articulando las sociedades en un sistema global. 
Se redefinen las relaciones de poder y se prioriza la dimensión económica para mejorar la competitividad, siguiendo un modelo de desarrollo basado en la «quimera del crecimiento» (Folch, 2011). El mismo concepto de bienestar se ha visto reducido únicamente a su sentido material, dejando al margen su significado más auténtico relacionado con la calidad de vida o el «buen vivir»(Acosta, 2013; Hessel \& Morin, 2012).

De esa forma las lógicas del mercado global han ido afectando a los procesos territoriales, generando desequilibrios sociales y medioambientales a diferentes escalas: los territorios ocupan un rol económico y una posición en el mercado global, de manera que la producción y los capitales tienden a localizarse en algunas áreas y salir de otras, generando de esta forma una fuerte dependencia respecto al transporte (Fernández Durán \& González Reyes, 2014).

Pero esta polarización social y económica no se produce solo entre territorios, sino que también se ve reflejada dentro del ámbito urbano, entre las zonas ricas y los barrios centrales y las zonas desfavorecidas y marginales, físicamente desconectadas o aisladas, convirtiendo las ciudades en «ámbitos disputados». De la misma forma esta desigualdad se evidencia a escala global entre los países ricos, donde se acumula la riqueza, y el resto del mundo, que sufre cada vez más una precarización de sus condiciones de vida (del Moral Ituarte, 2014; Naredo, 2004).

El fuerte impacto ambiental, la segregación social a diferentes escalas y la ineficiencia económica derivada de los altos costes energéticos están entre las principales consecuencias negativas de las dinámicas de la economía global, así como de los consecuentes fenómenos de polarización de los territorios y su fragmentación por la creciente expansión urbana que sigue un modelo de ciudad difusa, dispersa y desordenada, que separa piezas y funciones urbanas (Fariña Tojo \& Manuel Naredo, 2010). Es en las ciudades, y sobre todo en las grandes áreas metropolitanas, donde se generan los espacios de consumo y de creación de insostenibilidad más impactantes del planeta y en donde las decisiones reales sobre lo que ocurre en su entorno escapan cada vez más a la capacidad de control de los ciudadanos (Sassen, 1991; Verdaguer Viana-Cárdenas, 2002b).

Pero al mismo tiempo las ciudades tienen un papel crucial para invertir esta tendencia. Para ello todo proceso de trasformación urbana y territorial tiene que ser planificado. El planeamiento como disciplina técnica del urbanismo es un logro fundamental por ser un instrumento legal que, con sus herramientas, metodologías y formación específica, ofrece las bases para una ordenación y gestión sostenibles del territorio. Sin embargo, necesita una reformulación para recuperar su vocación de herramienta a servicio de los ciudadanos. En este sentido resulta preciso invertir los procesos de decisión de «arriba-abajo» para crear formas de intervención de «abajo-arriba» que rescaten al ciudadano de la condición de consumidor/usuario y le devuelvan su papel de protagonista en la construcción cultural y colectiva de la ciudad (cfr. Verdaguer Viana-Cárdenas, 2002a, p. 17).

\subsection{Respuestas a las lógicas de crecimiento de las ciudades territorializadoras}

La nueva ciudad territorializadora desborda, superando el clásico limite rural-urbano. La ciudad ya no es sinónimo de compacidad, de mezcla de usos y diversidad 
(Monclús, 1998), sino que traspasa sus límites, difuminándose con el campo, desestructurando los sistemas de su entorno y ocupando áreas cada vez más extensas (López Trigal \& Relea Fernández, 2001). La extrema dispersión actual de usos constituye uno de los principales factores de deterioro del medio ambiente y de fragmentación del territorio. Paulatinamente, el espacio físico continuo va perdiendo importancia ante el espacio abstracto discontinuo de las grandes redes globales, soportadas por unas articulaciones cada vez más densas y espesas, superpuestas. Los nodos (las grandes ciudades) tienen en este modelo una importancia fundamental debido a su papel económico en las escalas del mercado global.

El modelo de las sociedades urbano-industriales actuales se basa en un modelo de «ciclo abierto», constituido por núcleos centralizados de consumo/producción. En este modelo materiales y energía se absorben de forma cada vez más intensa, superando las capacidades de regeneración de recursos por parte de los ecosistemas y las capacidades naturales de asimilación de los residuos producidos por la actividad humana (Requejo Liberal, 2011). Pero el elemento esencial sobre el cual se basa todo este modelo es la enorme cantidad de "recorridos horizontales de los recursos de agua, alimentos, electricidad y combustibles, capaces de explotar otros ecosistemas lejanos y provocar importantes desequilibrios territoriales a escala planetaria" (Higueras, 2013, p. 70).

Frente a este modelo de "sistema abierto", que ya en diferentes ocasiones ha demostrado su insostenibilidad e inviabilidad a medio plazo, diferentes autores proponen otro tipo de modelo mediante el cual cada población pueda procurar, ordenar y obtener de su territorio la base fundamental de su sustento en ciclos semi-abiertos (v. gr. Requejo Liberal, 2011). Se trata de apostar por un modelo de desarrollo de tipo prevalentemente endógeno, basado en una diversificación productiva y donde las conexiones en red permitan el intercambio necesario para que los sistemas sean capaces de completar la propia autosuficiencia. "En la medida que se tenga más autosuficiencia en las múltiples capas de la gestión de nuestro hábitat, se tendrá más capacidad de decisión sobre qué tipo de espacio habitable y de ritmo vital queremos desarrollar" (Guallart, 2012, p. 21).

Para ello hay que buscar una optimización, dentro del propio territorio, de las posibilidades de obtención de satisfactores que atiendan a las verdaderas necesidades humanas (Max Neef et al., 1994) recurriendo al exterior para aquellos bienes y servicios que no sean suficientes o no se puedan obtener con medios propios; además, se necesita volver a recuperar el antiguo equilibrio entre territorio y hombre, desarrollando la capacidad de integrar las actividades humanas en las lógicas y procesos ecosistémicos (Requejo Liberal, 2011).

Por último, es necesario responder a las crecientes demandas de innovación democrática, otorgando a la ciudadanía un papel protagonista en la toma de decisiones, superando las formas tradicionales de democracia representativa (De Manuel Jerez, 2010; Requejo Liberal, 2011; Romero, 2011). Dicho de otra forma, se trata de dotar a nuestros territorios de una mayor resiliencia para lograr el equilibrio dinámico en los momentos de crisis (Ojeda Rivera \& Villa Díaz, 2008), apoyándose sobre todo en la capacidad de respuesta y en la implicación de las comunidades (en 
la escala del territorio) y de las unidades vecinales (en la escala urbana) para la transformación ecológica del territorio y de la ciudad.

En este sentido resulta imprescindible dejar a un lado la concepción del territorio como mero soporte físico de la actividad humana: el territorio es recurso valioso y no renovable, y a la vez conjunto complejo de historia, cultura, memoria colectiva, identidad; es espacio de tensión, en donde existe una contraposición de muchos intereses diversificados, y por eso necesita una mirada que sea global, compleja, holística y, sobre todo, compartida, para recuperar las diferentes perspectivas que nos ayuden a comprender esa complejidad.

Así, hay que complejizar no sólo los procesos y modos de planificación y gestión ${ }^{1}$, sino también la construcción misma del conocimiento sobre el hábitat. Nuestro actual sistema de conocimiento científico está de hecho fundamentalmente basado en el paradigma de la simplicidad que, con sus principios de separación, reducción y abstracción, por una parte ha posibilitado desarrollos extraordinarios en distintos campos del saber, aunque por otra ha aislado el saber entre campos de conocimiento, dejando en zonas de sombra todas las cuestiones transversales a los límites disciplinarios, siendo así difícilmente encuadrables (López Medina, 2012; Morin, 1994).

Por el contrario, todas las situaciones en que nos enfrentamos en el hábitat poseen componentes complejos, inabordables desde un solo punto de vista", así que requieren que "el flujo de saberes sea abierto y sujeto a una permanente incertidumbre que deje espacio a la introducción de nuevas relaciones complejas (Lucca, 2017, pp. 17-18).

Por ello es preciso reformular nuestro paradigma del pensamiento refundando el mismo desde el paradigma de la complejidad: si la disciplina es unidad parcelaria del conocimiento, la transdisciplina es constructo complementario de la misma (Lucca, 2009), que permite diluir las barreras disciplinares, asumir la incertidumbre y la imposibilidad de un conocimiento completo y, sobre todo, reconstruir el vínculo entre sujeto y objeto (Morin, 1994). Es necesario reintegrar al sujeto en la producción del conocimiento (Ibañez, 1992), que constituye en sí misma un hecho sociocultural que no puede ser considerado sólo como patrimonio exclusivo de la esfera técnico-institucional: el conocimiento experto tiene una doble vertiente, incluyendo tanto el experto sectorial (especialista) como el experto vivencial (el afectado directo de la situación a estudiar).

Por todo ello es preciso que la responsabilidad de tomar las principales decisiones que afectan a la organización territorial recaiga en el conjunto de la sociedad, a partir de la creación de espacios de participación y concertación donde sea posible alcanzar la construcción de dicho conocimiento experto, en su doble vertiente. En ese sentido y como se verá más adelante, muchos autores definen el proceso participativo como una construcción cíclica del conocimiento, durante la cual se suceden momentos de apertura y de cierre (síntesis o sistematización) que van marcando su «respiración» a lo largo del tiempo, según un esquema en espiral.

1 No sólo con el cambio de escala en la planificación y gestión del territorio, sino también con la necesidad de superar las políticas sectoriales y fragmentarias, a menudo incoherentes entre sí, y que reflejan una vez más los límites de una forma de conocimiento de tipo disciplinario. 
Así, se quiere aquí destacar la importancia del factor social, sobre todo al individuar a los ciudadanos como la solución para construir creativa y colectivamente nuevas y enriquecedoras formas de vida, más conectadas a lo comunitario, y al aceptar los límites biológicos del planeta: lo transicional pasa por lo social, es decir, por comunidades activas que generan ideas e intercambian información, para construir en el territorio proyectos comunes alternativos hacia la sostenibilidad.

Y esto se hace todavía más evidente en las cuestiones ambientales y territoriales de carácter complejo (Villasante, 2006): en contextos de incertidumbre y pluralidad de perspectivas legítimas de los distintos actores sociales, económicos e institucionales implicados, los actores locales "pueden imaginar soluciones y reformular problemas de manera que los expertos acreditados oficialmente, con la mejor voluntad posible, no encuentran ortodoxas dentro de su propio paradigma profesional" (Del Moral Ituarte \& Pedregal Mateos, 2002, p. 128).

\subsection{De usuarios a ciudadanos de la Polis}

Aunque existan cada vez más experiencias participativas que demuestran que la participación ciudadana es vista hoy en día como una exigencia democrática, se observa en general, entre los diferentes actores urbanos involucrados, una falta de cultura participativa madura que afecta al alcance efectivo de la participación, ya sea esto por una falta de acierto metodológico o por una baja coherencia política del planteamiento (Red CIMAS, 2015). Pero tras este hecho existen también otras motivaciones relacionadas más bien con la dimensión cultural que ejerce de motor en la forma de actuar de nuestras sociedades, influyendo en ella y justificándola. Martín Recio (1998) y Alberich (2008) identifican tres «enfermedades sociales»-la dependencia interpersonal, la compartimentación permanente y la delegación permanente- que provocan una falta de participación generalizada en sociedades individualistas como son las sociedades actuales del mundo occidentalizado.

Por el contrario, es necesario que el ciudadano recupere su papel de protagonista de la vida pública, de ciudadano de la $\pi$ ó $\lambda \varsigma^{2}$. Si es verdad que "para cambiar el rumbo del mundo, es preciso cambiar los mecanismos de gestión” (De Manuel Jerez, 2006, p. 89), la solución no se encuentra ni en la mano invisible que regula los mercados, ni en los técnicos y los políticos profesionales que por sí solos no tienen capacidad de formular políticas transformadoras, entre otras cosas porque:

[...] lo que está en juego es un modelo de civilización, una forma de entender la vida que afecta a los comportamientos cotidianos, y esta tarea no es posible acometerla sin poner en marcha procesos que nos impliquen a todos. Tenemos que cambiar de valores para cambiar de políticas (íbid.).

2 Más allá de las fuertes limitaciones existentes en la antigua Grecia democrática sobre qué categorías entraban en la definición de ciudadano de hecho y de derecho, todo ciudadano considerado como tal tenía derecho y deber de participar directamente a la vida de la ciudad, pudiendo ser designado para ocupar cargos públicos de diferente naturaleza; y aquellos que renunciaban a ocuparse de los asuntos públicos

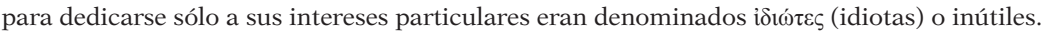


No se trata, sin embargo, de anular el papel de profesionales y especialistas en el proceso de aprendizaje y análisis sobre la realidad (Donadei, 2017; Red CIMAS, 2015), ni de plantearse una sociedad que funcione sin representantes y sólo por participación directa (Alberich et al., 2009), sino de profundizar en la democracia pasando de un modelo de tipo representativo, actualmente en crisis, a nuevas fórmulas complejas que permitan generar espacios en donde las decisiones se tomen conjuntamente entre administraciones, técnicos y ciudadanos de forma equitativa (Encina \& Rosa, 2004) y en el respeto a los principios ecológicos.

\subsection{La participación desde el paradigma de la PGSH}

En la Producción y Gestión Social del Hábitat (PGSH) se identifican tres grupos de actores con relaciones dialógicas y recursivas entre sí, generalmente conflictivas por tener intereses enfrentados y distinto poder en la toma de decisiones:

1. Los responsables políticos de la administración pública, en todos sus niveles, a quienes corresponde aprobar programas de hábitat y tomar decisiones sobre diferentes ámbitos (económicos, sociales, culturales, etc.), velando siempre por el interés general.

2. Los técnicos y los científicos, al servicio público o privado, encargados de asesorar y gestionar los programas de hábitat.

3. Los ciudadanos, que presentan su gran diversificación de necesidades en materia de hábitat.

Por otro lado, a menudo resulta adecuado introducir uno o más grupos de actores desglosando los grupos anteriores. Así, el triángulo original se puede desvirtuar al desglosar, por ejemplo, la categoría de ciudadanos en dos nuevos actores: el sector económico, constituido por aquellas empresas vinculadas al Hábitat (promotoras, constructoras, empresas suministradoras, etc.) y otras organizaciones y entidades que constituyen la sociedad civil (Figura 1).

La construcción de la ciudad ha estado siempre marcada por una variable relación entre estos grandes grupos de actores: si por una parte el arquitecto habitualmente ha trabajado para el «príncipe» y para el «mercader», por otra el pueblo ha construido el resto de la ciudad por sí mismo (De Manuel Jerez, 2006; Sassen, 2011; Verdaguer Viana-Cárdenas, 2002b).

Así, la participación se entiende como la redistribución equitativa del poder de decisión en todos los procesos que tienden a la transformación social del hábitat social (Encina \& Rosa, 2004), para que los sectores destinatarios de los procesos de transformación no se queden atrapados en su tradicional papel pasivo de receptores del cambio (Pelli, 2007). De este modo la responsabilidad de tomar las principales decisiones que afectan a la organización del hábitat recae en el conjunto de la sociedad a partir de la creación de espacios de participación y concertación donde políticos, técnicos y ciudadanos están llamados a compartir esa voluntad de iniciar un largo proceso de concertación, co-creación y cooperación, mediante un proceso de aprendizaje colectivo que llegue a construir el "triángulo equilátero de la gestión social del hábitat” (De Manuel Jerez, 2010, p. 18). 
La construcción equilátera del triángulo puede partir desde la iniciativa de cualquiera de estos tres grupos de actores para empezar construyendo un primer lado (la base): puede ser técnico-vecinal, político-vecinal o técnico-política (Figura 1). Una vez construida esta base, se trabajará para involucrar al vértice que falta: cuantos más actores se involucren en la formulación del problema, mayor será la incidencia social real.

\section{Figura 1. Activación de cambios desde las tres categorías de actores y posible des- glose de los actores socio-económicos}

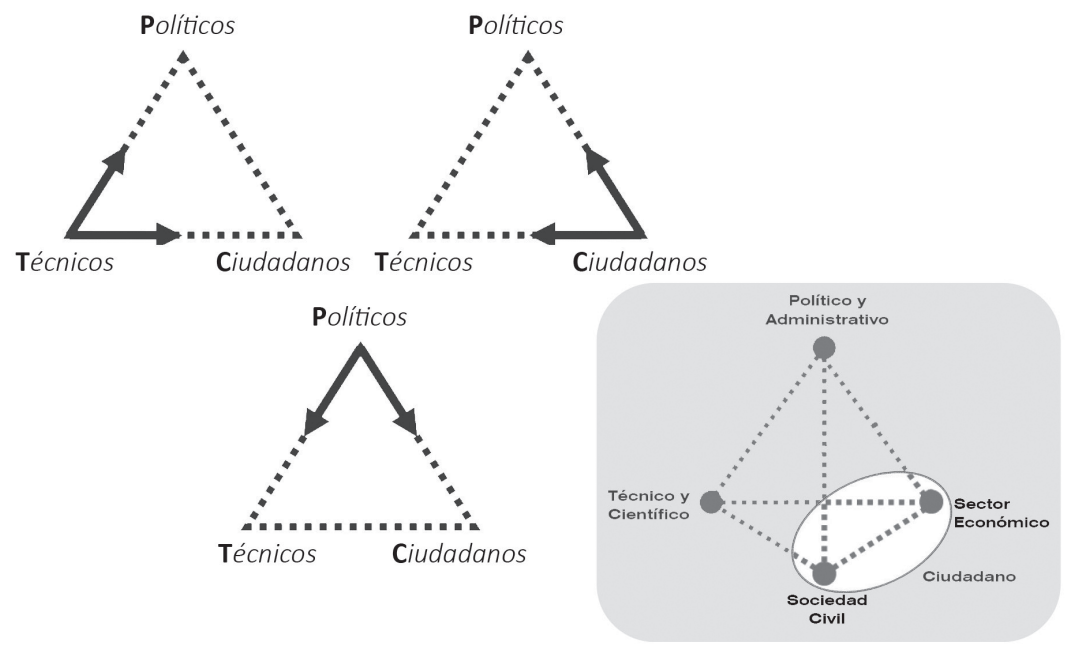

Fuente: Elaboración propia a partir de Manuel Jerez (2010) y Donadei (2017)

En este contexto se defiende el uso de la Investigación-Acción Participativa (IAP) como base metodológica para poder acompañar a los distintos grupos de actores involucrados en el aliento de procesos de participación que permitan la construcción colectiva del conocimiento y el diseño participado de estrategias complejas de intervención integral que conviertan los barrios, ciudades y territorios en piezas clave movilizadoras de la transición ecológica a diferentes escalas. La IAP, entendida como proceso de investigación social y a la vez de intervención social -y con cierta dosis de compromiso social (desde las posturas más militantes a otras de implicación afectiva- (Alberich, 2008; Montañes Serrano \& Martín Gutierrez, 2017; Villasante \& Montañes, 2002), trata de unir el tiempo del estudio con el tiempo de la acción para la consecución de los resultados en términos de transformación de una situación colectiva, estimulando la participación de la ciudadanía en todas las fases de la investigación-acción. De la misma forma, trata de rescatar la validez de los saberes y habilidades populares fruto de la interacción con los otros, con el medioambiente y con la propia cultura de pertenencia y con la esfera institucional.

De esta forma la IAP propicia el diálogo como mecanismo con el que crear procesos donde los sujetos afectados aporten, tras la reflexión, soluciones a sus pro- 
blemas (Álvarez \& Álvarez, 2007). Así, se entiende la participación ciudadana como un proceso de naturaleza cíclica e incremental, una «espiral espacio-temporal» de intercambio y construcción colectiva del conocimiento que empieza con la toma de conciencia de la necesidad de afrontar un problema de hábitat por parte de una comunidad. En ella cada ciclo va ganando en complejidad organizativa y en número de actores (Rosa, Saavedra y Hernández 2008; Rosa \& Encina, 2005) para llegar progresivamente a definir e implementar estrategias de acción y que viene alentada (y no conducida o dirigida) por expertos metodológicos (Encina \& Ávila, 2010).

Se construye conocimiento en y para la acción transformadora, en una estrecha colaboración entre la gente y los técnicos que les prestan apoyo, pasando por sucesivos grados de reflexividad en la elaboración de la estrategia de necesidades, o en la toma progresiva de decisiones (Figura 2).

\section{Figura 2. Desarrollo espiral de las metodologías participativas}

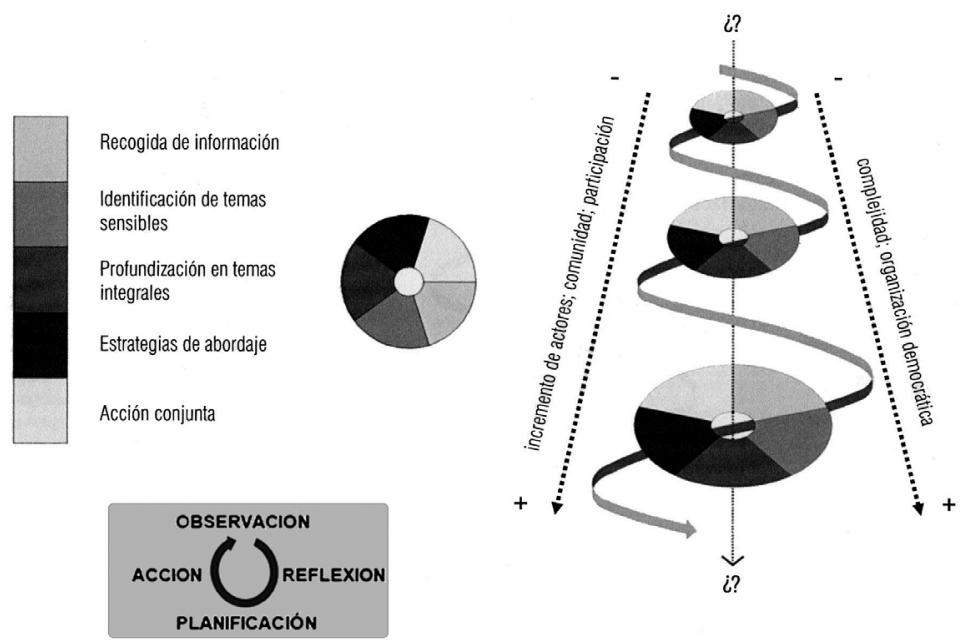

Fuente: Rosa, Saavedra y Hernández (2008)

Son tres las condiciones previas indispensables para la activación de procesos de participación realmente eficaces: el querer, el poder y el saber participar (Encina \& Ávila, 2010; Montañés Serrano, 2008):

[un] querer que concierne las motivaciones, un poder relativo a los cauces y un saber referido a las habilidades. Pero [...] a la luz del paradigma de la complejidad, no entendidas como premisas independientes y apriorísticas sino como elementos vinculados en una misma estrategia: un querer más motivado a partir de la existencia de cauces y habilidades; unos cauces más demandados a partir de la motivación y las capacidades; y un saber más alentado a partir de la apertura de cauces y el impulso de las motivaciones (López Medina, 2012, p. 166).

Así, el «querer participar» se relaciona con el sentimiento de pertenencia que da las motivaciones para involucrarse en el proceso: para ello es necesaria la aplicación de 
estrategias de identificación social que surjan desde la cultura popular y que trabajen con las identificaciones y afectividades de los grupos sociales implicados (Figura 3).

\section{Figura 3. De usuario a ciudadano}

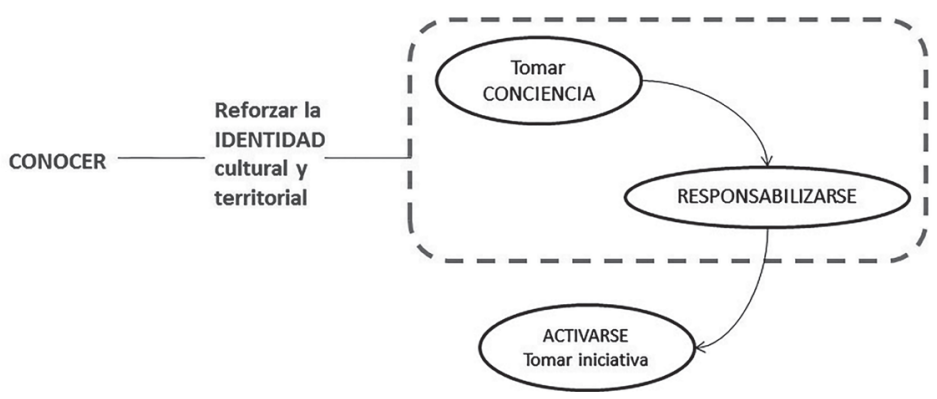

Fuente: Donadei (2017)

El «saber participar» está relacionado con la toma de conciencia del significado de "participar», es decir, que cada ciudadano y colectivo involucrado interiorice el hecho de que al propio interés personal se antepone el interés colectivo (y en el respeto de los intereses ambientales). Para activar esta transición de sujetos individuales a sujetos colectivos es necesario trabajar metodológicamente para provocar no sólo cambios organizacionales, sino también intercambios a nivel simbólico y en la gramática cultural, así como cambios en el sistema de reglas que estructuran las relaciones e interacciones sociales (Rosa \& Encina, 2004).

Por último, el "poder participar» se refiere a la necesidad de que existan las circunstancias idóneas para que la participación sea posible. Para ello se requiere la adecuación de espacios y tiempos que permitan a los diferentes actores sociales involucrarse a la hora de tomar parte en el proceso. Eso implica superar las actuales y enrevesadas formas y estructuras administrativas, dando cabida a nuevas y renovadas formas de urbanismo basadas en metodologías más dinámicas y flexibles; pero además, se precisa una voluntad política real para ceder poder al ciudadano.

Figura 4. La escalera de la participación

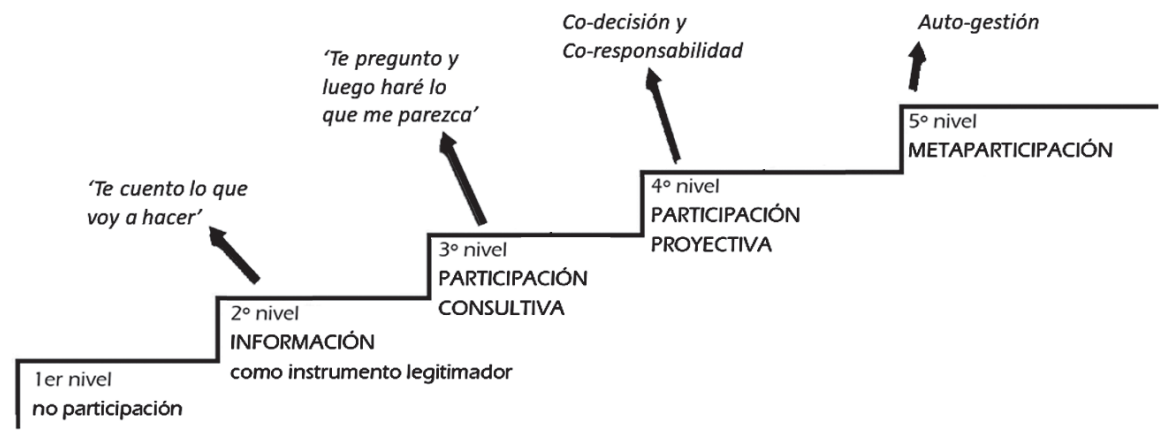

Fuente: Elaboración propia a partir de López Medina (2012) y RED CIMAS (2015) 
Por otro lado, hay que considerar que se trata de una apertura a la implicación de otros actores en un proceso al que a priori no tienen acceso acceso (Arnanz Monreal, 2018; López Medina, 2012). Por lo tanto, es necesario aclarar también cuál es el grado de apertura e implicación oportuno para considerar que un proceso participativo sea realmente eficaz. Son muchos los autores ${ }^{3}$ que han propuesto la imagen de una pirámide o escalera para señalar los diferentes gradientes de participación: en todos ellos lo que resulta evidente es que el nivel más bajo está ocupado por modelos de gestión no participativos hasta llegar a los modelos más emancipadores, en donde la ciudadanía asume un papel activo en la toma de decisiones, junto a técnicos y políticos.

Todos estos modelos se pueden resumir en un esquema de cinco peldaños (Figura 4). En el nivel más bajo estaría la gestión directa sin participación, en donde las decisiones se toman en las instancias de poder que suponen un cargo político o rol técnico. En el segundo nivel se encontrarían las fórmulas que prevén la simple información como instrumento legitimador: "sin embargo, esto no puede considerarse participación sino un requisito previo [...] para que pueda haber participación” en cuanto "la información favorece la toma de conciencia crítica, la creación de opinión y la trasparencia” (Red CIMAS, 2015, p. 44). Un tercer nivel está caracterizado por la consulta de opinión, muy característico de muchos de los procesos de redacción de documentos urbanísticos de diferente naturaleza y cuyo único objetivo es a menudo darle un cierto aire participativo al proceso, sobre todo por el hecho de que en la mayoría de los casos los resultados se convierten en meras ratificaciones de las propuestas por medio de matizaciones pero sin modificaciones sustanciales, o se recogen en algún documento complementario que no tiene carácter vinculante y cuya aplicación solo depende del voluntarismo político.

Los últimos dos niveles tienen que ver con la implicación real de la ciudadanía en la toma de decisiones, ejerciendo de este modo, una vez que ha sido informada, consultada y escuchada, el derecho a ser sujeto activo de los procesos de transformación del hábitat. Si en el cuarto nivel se asume como principio la co-decisión y la co-responsabilidad, y en él la ciudadanía comparte la gestión de los procesos de trasformación junto a políticos y técnicos, es el último nivel el que supone realmente un salto cualitativo enorme en el entendimiento de la participación, necesitando un alto grado de madurez por parte tanto de la ciudadanía como del resto de grupos de actores involucrados.

A este nivel corresponden la auto-organización y la auto-gestión, con las cuales la ciudadanía organizada se hace cargo de la gestión de alguna parcela de la vida pública (López Medina, 2012; Red CIMAS, 2015). En este sentido la participación adquiere un grado mayor de complejidad en cuanto desplaza:

[...] el acento de los procesos institucionalizados a los procesos de la vida cotidiana de la población como fuente de mecanismos de autogestión a los que alimentar y fortalecer desde lo público. [...] En este sentido, el hábitat se construye de forma cotidiana (López Medina, 2012, p. 168).

3 La primera autora que introdujo la figura metafórica de la escalera fue Sherry Arnstein, trabajadora social estadounidense. 


\section{Discusión y conclusiones}

La crisis ecológica en acto demuestra que es necesario replantearse el modelo, pasando por una revolución cultural que deberá desembocar en una refundación de lo político (Latouche, 2009). Si un primer paso para avanzar hacia la «coevolución» ${ }^{4}$ entre los sistemas humanos y ecológicos es la convergencia entre teoría y práctica en la integración de principios de sostenibilidad ambiental en las políticas y los instrumentos legales del urbanismo y de la ordenación y gestión del territorio, el segundo paso sería que los principios ecológicos no se conviertan en un nuevo dogma que sustituya la capacidad de decisión colectiva de una comunidad (Lucca, 2015; Verdaguer VianaCárdenas, 2002b), sino que esos principios sean asimilados por su grado de madurez.

En este sentido, los ciudadanos resultan ser los sujetos activos de este cambio en cuanto son ellos los que definen la territorialidad a través de sus prácticas cotidianas (Bonora, 2006). En contraposición a la actitud dominante de una teórica participación, que a menudo se concreta en eventos y acciones puntuales (e impulsadas por las diferentes administraciones), es necesario apostar por una apropiación real, por parte de los ciudadanos, del nuevo metabolismo territorial y urbano, reforzado al mismo tiempo con un mayor grado de implicación y responsabilidad por parte de las comunidades en las cuestiones ambientales que afectan a su entorno y a sus paisajes fundantes.

Así, el gran reto a largo plazo es que la participación ciudadana se convierta en un hábito, un laboratorio de experimentación constante en el cual adaptar lenguajes, instrumentos y métodos propios de las diferentes disciplinas para generar estrategias complejas y colectivas de intervención sobre el hábitat, orientadas hacia la transición ecológica. Entendida de esta manera, la participación no sólo puede ayudar a la transformación de las condiciones materiales de un determinado ámbito, sino que, a partir de un trabajo profundo que requiere grandes esfuerzos por parte de todos los grupos de actores involucrados, permite generar un proceso en el que ciudadanos, políticos y técnicos maduren colectivamente. Acompañándola en este camino, la ciudadanía activa será capaz de tomar las riendas de su lugar de vida.

\section{Referencias}

Acosta, A. (2013). El buen vivir. Sumak Kawsay, una oportunidad para imaginar otros mundos. America Latina: Icaria-Antrazyt.

Alberich, T. (2008). IAP, redes y mapas sociales: desde la investigación a la intervención social. Portularia: Revista de Trabajo Social, VII(1), 131-151. (http://bit.ly/2snoyHT) (2018-11-14).

Alberich, T., et al. (2009). Metodologías participativas. Manual. Madrid: CIMAS. (http://bit.ly/2RpwR4J) (2018-12-03).

Álvarez, C., \& Álvarez, A. (2007). La Investigación Acción Participativa como instrumento para la resolución de problemas sociales. Anuario, 30, 227-252. (http://bit.ly/2AJyBf3) (201901-03).

4 Por coevolución se entiende el proceso de interacción recíproca en el tiempo entre los sistemas socio-culturales y los sistemas biofísicos, que mantienen una estrecha interacción condicionándose mutuamente por mecanismos de retroalimentación y adaptación (Norgaard, 1984). Si bien a lo largo de la historia las sociedades se han beneficiado de este tipo de procesos, el modelo económico dominante ha roto este equilibrio presionando al sistema ecológico más allá de su potencial de coevolución. 
Arnanz Monreal, L. (2018). Metodologías de la democracia participativa en el ámbito de las políticas públicas locales: hacia un sistema de gestión participativo. En E. Santos (Ed.), Metodologías participativas y democracias transformadoras (pp. 31-38). Madrid: CIMAS.

Bayon, D., Flipo, F., \& Schneider, F. (2011). Decrecimiento: 10 preguntas para comprenderlo y debatirlo. Intercención Cultural.

Benevolo, L. (1979). Introducción a la arquitectura. Madrid: Blume.

Bonora, P. (2006). Percorsi della socialità e della partecipazione negli spazi deterritorializzati dell 'urbano. Inchiesta, 152, 3-5.

Cambil Medina, E., et al. (2006). Líneas de investigación en ADICI: Diseño de procesos de Producción Social del Hábitat. En II Jornada de Investigación en Arquitectura y Urbanismo.Escuela De Arquitectura Del Vallès, Sant Cugat Del Vallès. (http://bit.ly/2SQGPZF) (2018-11-14).

Caravaca Llamas, C. (2012). Más decrecimiento y menos Prozac. El decrecimiento como alternativa económica y social a la crisis actual. La Razón Histórica, 18, 81-101. (https://www. revistalarazonhistorica.com/18-7/)

Castiblanco, C. (2008). La Economía Ecológica: Una Disciplina En Busca De Autor. Gestión y Ambiente, 10(3), 07-22. (http://bit.ly/2Mabmz0) (2018-11-14).

Common, M., \& Stagl, S. (2008). Introducción a la economía ecológica. Barcelona: Reverté.

De Manuel Jerez, E. (2006). Participar para recuperar la polis. En Educación de Adultos y Participación (pp. 89-102). Sevilla: Unilco.

De Manuel Jerez, E. (2010). Construyendo triángulos para la gestión social del hábitat. Hábitat y Sociedad, 1, 13-37. http://dx.doi.org/10.12795/HabitatySociedad.2010.i1.02.

Del Moral Ituarte, L. (2014). Crisis del capitalismo global. Desarrollo y medio ambiente. Documents d'Anàlisi Geogràfica, 59(1), 77-103. https://doi.org/10.5565/rev/dag.36

Del Moral Ituarte, L., \& Pedregal Mateos, B. (2002). Nuevos planteamientos científicos y participación ciudadana en la resolución de conflictos ambientales. Documents d'Anàlisi Geogràfica, 41, 121-134. (http://bit.ly/2TMNTGR) (2018-12-03).

Díaz Rodríguez, P., \& Rodríguez Darias, A.J. (2012). Fundamentos del paradigma ecológico en las ciencias sociales. Pasos, 10(1), 167-172. http://doi.org/10.25145/j.pasos.2012.10.016

Donadei, M. (2017). Conservación de la naturaleza y bienestar humano: el papel de la participación ciudadana en la transición socio-ecológica de la aglomeración urbana de Sevilla (Tesis Doctoral). ETSA Sevilla, España.

Emmott, S. (2013). Diez mil millones. Barcelona: Anagrama.

Encina, J., \& Ávila, M.Á. (2010). El ilusionismo social: más allá de la última frontera metodológica. En J. Encina, M.Á. Ávila, \& B. Lourenço, (Ed.), Las culturas populares (pp. 73-104). Sevilla: Atrapasueños.

Encina, J., \& Rosa, M. (2004). Democracias participativas desde la práxis social. En J. Encina, M. Rosa, M.Á. Ávila, \& M. Fernández (Eds.), Democracias participativas e intervención social comunitaria desde Andalucía (pp. 13-46). Sevilla: ACSUR-Andalucía, Atrapasueños Editorial, UPO, Consejería de Asuntos Sociales-Junta de Andalucía.

Fariña Tojo, J., \& Manuel Naredo, J. (2010). Libro blanco de la sostenibilidad en el planeamiento urbanístico español. España: Ministerio de Vivienda. (http://bit.ly/2D7qt9D) (2018-10-26).

Fernández Durán, R., \& González Reyes, L. (2014). En la espiral de la energía. Volumen 2: Colapso del capitalismo global y civilizatorio. Madrid: Libros en acción.

Folch, R. (2011). La quimera del crecimiento. La sostenibilidad en la era postindustrial. Barcelona: RBA Libros.

Freire, J. (2009). Urbanismo emergente: ciudad, tecnología e innovación social [Emerging urban planning: city, technology and social innovation*. En Paisajes Domésticos / Domestic Landascapes, Vol. 4 (pp. 18-27). Madrid: SEPES.

García Montes, N. (2018). Desarrollo urbano, crisis ecológica e importancia epistemológica de la percepción ciudadana. En E. Santos (Ed.), Metodologías participativas y democracias transformadoras (pp. 31-38). Madrid: CIMAS. 
Georgescu-Roegen, N. (1971). The Entropy Law and the Economic Process. Cambridge: Harvard University Press.

González-Arriero, C. (2017) Urbanismo en transición. Producción y gestión social del Espacio Público (Tesis Doctoral). ETSA Sevilla, España.

Guallart, V. (2012). La ciudad autosuficiente. Barcelona: RBA Libros.

Hessel, S., \& Morin, E. (2012). El camino de la esperanza: una llamada a la movilización cívica. Barcelona: Destino-Paidos.

Higueras, E. (2013). Urbanismo bioclimático. Barcelona: Gustavo Gili.

Ibañez, J. (1992). Más allá de la sociología. Teoría y práctica del grupo de discusión. Madrid: Siglo XXI editores.

Latouche, S. (2009). Pequeño tratado del decrecimiento sereno. Barcelona: Icaria.

López Medina, J.M. (2012). El diseño participativo en programas de rehabilitación de viviendas (Tesis Doctoral). ETSA Sevilla, España.

López Trigal, L., \& Relea Fernández, C.E. (2001). Ciudades y periferias sostenibles. Una revisión de conceptos y enfoques aplicados a España y Castilla y León. Polígonos, 11-12, 113-136. http://dx.doi.org/10.18002/pol.v0i11-12.523

Lovelock, J. (2006). La venganza de la tierra: la teoria Gaia y el futuro de la humanidad. Barcelona: Planeta.

Lucca, E. (2009). Interdisciplina. Cuadernos del Ambiente nº 1. Resistencia: Cospel.

Lucca, E. (2016). La gestión de los territorios. La cosecha escondida o la percepción ambiental de los espacios. Buenos Aires: Diseño editorial.

Lucca, E. (2017). Estrategias y metodologías de formación de equipos interdisciplinarios para trabajar en hábitat. Hábitat y sociedad, 10, 15-34. http://dx.doi.org/10.12795/HabitatySociedad.2017.i10.02

Marcellesi, F. (2010). Cooperación internacional y sostenibilidad. El Ecologista, 65, 30-31.

Martín Recio, S. (1998). Reflexiones y acciones para propiciar la participación comunitaria. En I Conferencia de Política Municipal. Madrid: IU Secretaría Federal de Política Federal de Entes Locales.

Max Neef, M., Elizalde, A., \& Hopenhayn, M. (1994). Desarrollo a escala humana: conceptos, aplicaciones y algunas reflexiones. Montevideo: Icaria/Nordan-Comunidad.

Meadows, D., Randers, J., \& Meadows, D. (2006). Los límites del crecimiento: 30 años después. Barcelona: Galaxia Gutenberg.

Monclús, F.J. (1998). Suburbanización y periurbanización. Ciudades anglosajonas y ciudades latinas. En F.J. Monclús (Ed.), La ciudad dispersa. Suburbanización y nuevas periferias (pp. 5-15). Barcelona: CCCB

Montañés Serrano, M. (2008). El cuadro semiótico y las posiciones complejas de la participación. Madrid: Cuadernos CIMAS. (http://bit.ly/2RK2qpr) (2019-01-06).

Montañes Serrano, M., \& Martín Gutiérrez, P. (2017). De la IAP a las Metodologías Sociopráxicas. Habitat y Sociedad, 10, 35-52. http://dx.doi.org/10.12795/HabitatySociedad.2017.i10.03

Morin, E. (1994). Introducción al pensamiento complejo. Madrid: Gedisa.

Naredo, J.M. (2000). Ciudades y crisis de civilización. Boletín CF+S, 15. (http://bit.ly/2RKLyPh) (2018-12-03).

Naredo, J.M. (2004). Diagnóstico sobre la sostenibilidad: la especie humana como patología terrestre. Archipiélago, 62, 13-24.

Naredo, J.M. (2011). Fundamentos de la Economía Ecológica. En F. Aguilera Klink \& V. Alcántara (Eds.), De la Economía Ambiental a la Economía Ecológica (pp. 231-252). Madrid: CIP-Ecosocial.

Norgaard, R.B. (1984). Coevolutionary development potential. Land Economics, 60(2), 160-173.

Ojeda Rivera, J.F., \& Villa Díaz, Á. (2008). La ciudad es cultura fronteriza en un mundo cambiante. En Ciudades, culturas y fronteras en un mundo en cambio. IX Coloquio de Geografía Urbana (pp. 284-301). Sevilla: Junta de Andalucía.

Pelli, V. (2007). Habitar, participar, pertenecer: acceder a la vivienda: incluirse en la sociedad. Buenos Aires: Nobuko. 
Pelli, V. (2010). La gestión de la producción social del hábitat. Hábitat y Sociedad, 1, 39-54. Retrieved from http://www.habitatysociedad.us.es/images/stories/N01/n01a02_la_gestion_del_ la_produccion_social_del_habitat.pdf

Red CIMAS. (2015). Metodologías participativas. Sociopraxis para la creatividad social. Madrid: Dextra Editorial.

Requejo Liberal, J. (2011). Territorio y energía: Orden mecánico versus orden orgánico. Hábitat y Sociedad, 2, 33-47. http://dx.doi.org/10.12795/HabitatySociedad.2011.i2.03

Romero, J. (2011). ¿Territorios inteligentes, sostenibles e inclusivos? Enseñanzas de la experiencia española. Hábitat y Sociedad, 2, 13-32. http://dx.doi.org/10.12795/HabitatySociedad.2011.i2.02

Rosa, M., \& Encina, J. (2004). Las culturas populares. Cuchara y Paso Atrás, (9), 9-20.

Rosa, M., \& Encina, J. (2005). Haciendo metodología al andar. En J. Encina, M. Á. Ávila, M. Fernández, \& M. Rosa (Eds.), Praxis participativas desde el medio rural (pp. 89-116). Madrid: IEPALA/CIMAS.

Rosa, M., Saavedra, L., \& Hernández, G. (2008). Fundamentos teóricos y metodológicos de la participación ciudadana. Conferencia en el Máster en Gestión Social del Hábitat (1 ${ }^{\mathrm{a}}$ Edición). US, Sevilla, España.

Sassen, S. (1991). The global city: New York, London, Tokyo. New Jersey: Princetown University Press.

Sassen, S. (2011). Open Source Urbanism. The New City Reader: A Newspaper of Public Space, 15. Retrieved from (http://bit.ly/2D7amc5) (2018-10-30).

Taibo, C. (2009). En defensa del decrecimiento. Sobre capitalismo, crisis y barbarie. Madrid: Catarata.

Verdaguer Viana-Cárdenas, C. (2002a). Ecologismo urbano y urbanismo ecológico: convergencia necesaria. El Ecologista, 34, 16-18.

Verdaguer Viana-Cárdenas, C. (2002b). El paisaje construido: una perspectiva ecológica. En Ó. Bermejo García, J.J. Laforet (Eds.), Ecología, una perspectiva actual (pp. 155-182). Telde: Real Sociedad Económica de Amigos del País de Gran Canaria.

Villasante, T.R. (2006). Desbordes creativos: estilos y estrategias para la transformación social. Madrid: La Catarata.

Villasante, T.R., \& Montañes, M. (2002). Algunos cambios de enfoque en las ciencias sociales. En J. Martí Olivé, M. Montañés, \& T. Villasante (Eds.), La investigación social participativa (pp. 13-28). Barcelona: El viejo topo. 